\title{
La humanización y su impacto en el sistema de gestión de la calidad del Hospital de Fontibón en Bogotá*
}

\section{Humanization and its impact on the quality management system of Fontibón's Hospital in Bogotá}

Recibido: 22 de enero de 2014 Revisado: 22 de marzo de 2014 Aceptado: 27 de junio de 2014

Ericka Trinidad Gaitan Gelve****

Universidad Santo Tomás - ICONTEG

Sonia Lu: Flores Gutiérrez****

Secretaría Distrital de Salud - Bogotá

\section{RESUMEN}

Dada la importancia de los servicios de urgencias de salud en Bogotá y el impacto que tiene el tema de la humanización en los sistemas de gestión de la calidad, en este trabajo se investiga la implementación de la humanización en el Hospital de Fontibón, según lo propuesto en el Plan de Desarrollo "Bogotá Humana 2012-2016", en el Plan Territorial de Salud "Bogotá Distrito Capital 2012 - 2016”, así como en el Programa de Humanización desarrollado por la Secretaría
Distrital de Salud. Los datos son obtenidos a través de entrevistas directas con la alta dirección y mediante encuestas al personal asistencial y a los usuarios. A partir de los resultados obtenidos se definen lineamientos estratégicos que se presentan a la Entidad, con el fin de materializarlos en acciones para mejorar la atención a los usuarios que acuden al servicio de urgencias.

Palabras clave: humanización de la salud, servicio de urgencias, sistema de gestión de la calidad.

* Artículo de investigación.

** Correspondencia: Ericka Trinidad Gaitán Gélvez. Microbióloga. Frimac S.A. Correo electrónico: ericka616@hotmail.com

*** Sonia Luz Flórez Gutiérrez. Administradora de Empresas. Secretaria Distrital de Salud- Bogotá. Correo electrónico: soflor11@hotmail.com 


\section{ABSTRACT}

Given the importance of emergency health services in Bogota and the impact of the humanization issue in the quality management systems, this paper investigates the implementation of humanization in the Hospital of Fontibon, as proposed in the development plan "Bogota Humana 2012-2016", in the territorial health plan "Bogotá Capital District 2012 - 2016" and in the Humanization Program developed by the District Health Department. Data are obtained through direct interviews with senior management and through surveys to caregivers and users. Strategic guidelines are obtained from the results and presented to the entity, to turn them in actions to improve care to users who come to the emergency service.

Keywords: Humanization of health, emergency services, quality management system.

\section{INTRODUCCIÓN}

La deshumanización de la salud se materializa cuando falta una atención médica adecuada al usuario y cuando éste solo es visto desde la perspectiva de la enfermedad y no desde el punto de integralidad, como miembro de una sociedad, que siente y vive (Parsons, 1966). La presente investigación explora la implementación de la humanización y su impacto en el sistema de gestión de la calidad del Hospital de Fontibón II Nivel E.S.E. Se plantean como objetivos: la elaboración de un diagnóstico de la humanización del servicio de urgencias del Hospital que permita identificar la efectividad de las acciones formuladas para humanizar este servicio y los posibles obstáculos que impidan su ejecución; identificar la percepción que tienen los usuarios de la humanización del servicio recibido en urgencias, bajo los criterios establecidos en el decálogo de humanización y formular lineamientos para crear una estrategia que integre lo contenido en el Plan Territorial en Salud y el Plan de Desarrollo Distrital con el sistema de gestión de la calidad del Hospital de Fontibón que pueda servir de modelo para los demás hospitales del Distrito.

\section{METODOLOGÍA}

Para identificar los elementos claves a intervenir y los obstáculos de la implementación del Programa de Humanización se realizan entrevistas al personal directivo; la información obtenida en estas entrevistas es la base para el diseño de las encuestas tanto al nivel asistencial (médicos, enfermeras, auxiliares) como a los pacientes del servicio de urgencias.

La determinación de la muestra se realiza teniendo en cuenta la planta de personal del Hospital y el número de usuarios atendidos en el servicio de urgencias en el año 2013 (meses de abril y mayo) según los históricos de atención del Hospital; se determinan las muestras con una seguridad del $95 \%$ y precisión del $5 \%$, obteniendo una muestra de 329 encuestas a usuarios y de 45 encuestas para la parte asistencial.

Los instrumentos estructurados fueron sometidos a una prueba piloto tanto en el caso de servidores públicos como en de los usuarios. Los datos obtenidos se sometieron a análisis estadístico mediante la aplicación de análisis univariado, bivariado y multivariado para los dos casos; además a la información de los usuarios se le aplicó el índice de componentes principales para determinar el índice de percepción sobre la humanización en el servicio de urgencias.

La información obtenida fue tabulada y se identificaron las categorías para el trabajo posterior y para excluir las variables no significativas, aquellas con variabilidad de $80 \%$ o más. Se utilizó el análisis univariado, el 
cual es un análisis básico en el que las características 0 propiedades han de medirse una a una (Universidad Nacional Abierta y a Distancia- UNAD, 2014a). Conforme a Jaime Fierro (2010), se utilizó la distribución de frecuencias que indica el número de casos que hay en cada categoría. A partir de dichos valores, se calculó el porcentaje respecto del total de observaciones.

El análisis bivariado permite determinar la existencia o no de relación entre dos variables, y permite ver como una variable influye en la otra (UNAD, 2014b). Los resultados se expresaron mediante gráficos de barras con porcentajes. Por otra parte el análisis multivariado permite analizar simultáneamente medidas múltiples de cada individuo u objeto sometido a investigación (UNAD, 2014c), es una técnica de aplicación en el análisis exploratorio de datos multidimensionales, donde las encuestas son el instrumento de medición de datos relevantes para una investigación; según Quaglino y Pagura (1998), este método ayuda a describir relaciones entre las características medidas, poniéndolas de manifiesto en un gráfico para su interpretación.

El Índice de componentes principales sirve para hallar las causas de la variabilidad de un conjunto de datos y ordenarlas según su importancia. Retiene aquellas características del conjunto de datos que contribuyen más a su varianza, manteniendo un orden de bajo nivel de los componentes principales e ignorando los de alto nivel (Giardina, 1967, p. 112); el concepto involucrado es que esos componentes de bajo orden, a veces contienen el aspecto "más importante" de la información.

\section{RESULTADOS Y DISCUSIÓN}

A continuación se presenta la información más relevante que resulta del procesamiento de los datos, en relación con el cumplimiento de los objetivos.

\section{Diagnnóstico de la Humanización en el Hospital Fontibón}

Mediante este diagnóstico se determinan el conocimiento de los servidores sobre la implementación del programa de Humanización y Calidad, la percepción de los usuarios sobre la prestación del servicio y sobre las acciones adoptadas por el Hospital, así como los obstáculos en el servicio de urgencias del Hospital, que identifican ambos actores.

\section{Servidores}

Como punto de partida para comprender la apropiación de los servidores de los enfoques y prácticas del Programa de Humanización, se preguntó por el conocimiento que tenían de las actividades de ese programa; los resultados se pueden ver en las Figuras 1, 2 y 3.

Los servidores más antiguos conocen mejor las actividades de humanización implementadas por la alta dirección (89\%) que los más recientes (69\%), lo cual concuerda con lo esperado.

Siendo el decálogo de Humanización uno de los temas que más deben manejar los servidores públicos se encontró que el $42 \%$ de los servidores que ingresaron antes del 2012 no lo recuerda mientras que si lo hace el $62 \%$ de quienes ingresaron del 2012 a la fecha; asimismo se encontró que el $86 \%$ de los servidores de la noche son los que le dan más importancia a las características del buen trato al paciente.

Por otra parte se encontró con respecto al sistema de gestión de la calidad que quienes más conocen las actividades son las personas del nivel asistencial con un $67 \%$ y los profesionales con un $54 \%$; quienes más han participado son los de la jornada de la mañana con un $50 \%$.

En cuanto a la adherencia a los planes operativos, el $77 \%$ de los servidores, manifestaron conocerlos, siendo el $67 \%$ personal de planta, lo que demuestra más su compromiso con la Entidad, que los contratistas. 
Fiǵura 1. Conocimiento de las actividades de humanización implementadas según el tiempo de vinculación

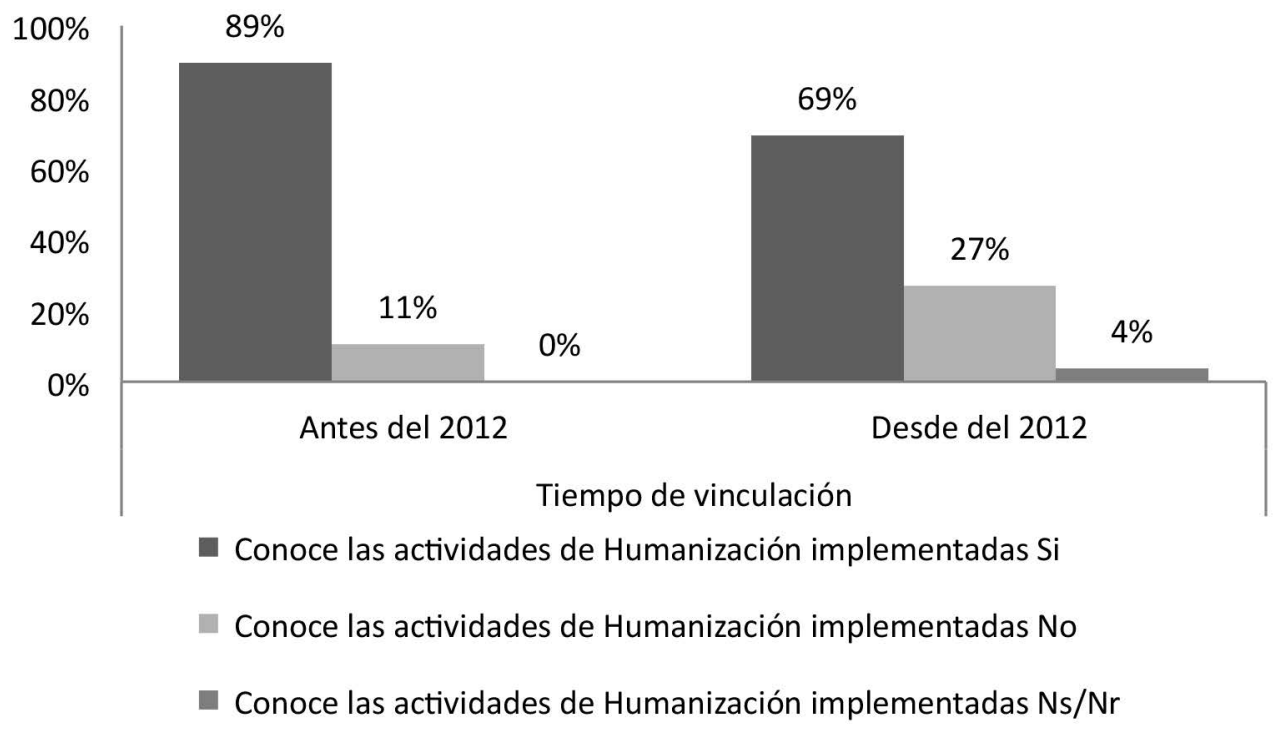

Fuente: elaboración propia.

Figura 2. Recuerda el decálogo de humanización según tiempo de vinculación
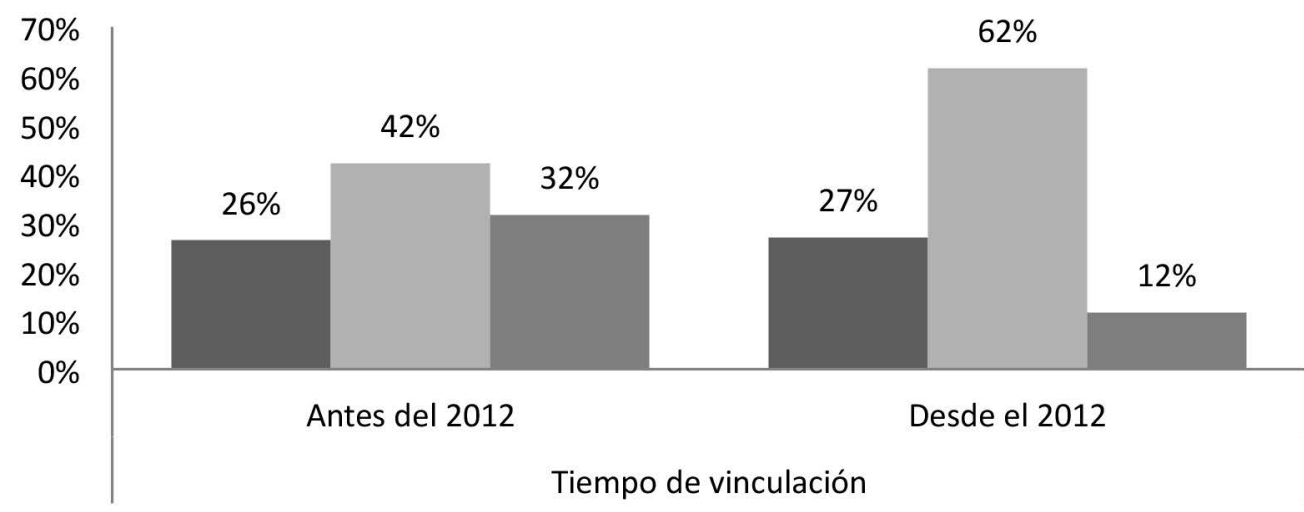

\footnotetext{
- Recuerda el decálogo de humanización si

Recuerda el decálogo de humanización no

Recuerda el decálogo de humanización $\mathrm{Ns} / \mathrm{Nr}^{*}$
}

Fuente: elaboración propia. 
Figura 3. Obstáculos para la implementación del programa de humanización según el tiempo de vinculación

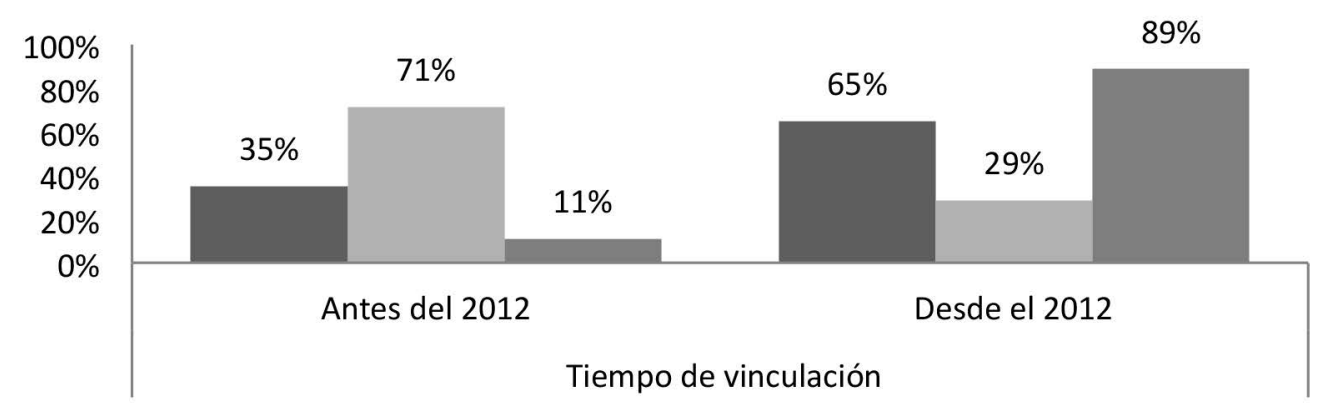

Estado de contingencia

Características del servicio de urgencias

Fallas de gestión administrativa y logística

Fuente: elaboración propia.

El análisis de la información de servidores fue realizado mediante Análisis de Correspondencias Múltiples (ACM), el cual permite identificar, según las características de las categorías, cuáles son los grupos que existen y suministra un gráfico de dichos grupos; para el caso de los servidores, se muestran en la Tabla 1.

Tabla 1. Caracterización por categorías de grupo para servidores (Clúster)

\begin{tabular}{|c|c|c|c|c|c|}
\hline \multicolumn{6}{|c|}{ Corte "a" del árbol en 2 Grupos (CLUSTERS) } \\
\hline \multicolumn{3}{|c|}{$\begin{array}{c}\text { Grupo: CLUSTER 1/2 } \\
\text { (Conteo: } 23 \text { - Porcentaje: 51.11) }\end{array}$} & \multicolumn{3}{|c|}{$\begin{array}{c}\text { Grupo: CLUSTER 2/2 } \\
\text { (Conteo: } 22 \text { - Porcentaje: 48.89) }\end{array}$} \\
\hline $\begin{array}{l}\text { Etiqueta de } \\
\text { Variable }\end{array}$ & $\begin{array}{l}\text { Características de } \\
\text { categorías }\end{array}$ & $\begin{array}{l}\text { Prueba } \\
\text { Valor }\end{array}$ & $\begin{array}{l}\text { Etiqueta de } \\
\text { Variable }\end{array}$ & $\begin{array}{l}\text { Características de } \\
\text { categorías }\end{array}$ & $\begin{array}{l}\text { Prueba } \\
\text { Valor }\end{array}$ \\
\hline $\begin{array}{l}\text { Características del } \\
\text { buen trato }\end{array}$ & $\begin{array}{l}\text { No características del } \\
\text { buen trato }\end{array}$ & 6,78 & $\begin{array}{l}\text { Características del } \\
\text { buen trato }\end{array}$ & $\begin{array}{l}\text { Si característica. del } \\
\text { buen trato }\end{array}$ & 6,78 \\
\hline $\begin{array}{l}\text { Valores y cualidades } \\
\text { necesarias }\end{array}$ & $\begin{array}{l}\text { No Valores y } \\
\text { cualidades necesarias }\end{array}$ & 6,41 & $\begin{array}{l}\text { Valores y cualidades } \\
\text { necesarias }\end{array}$ & $\begin{array}{l}\text { Si Valores y } \\
\text { cualidades necesarias }\end{array}$ & 6,41 \\
\hline $\begin{array}{l}\text { Necesidades del } \\
\text { paciente }\end{array}$ & $\begin{array}{l}\text { Si Necesidades del } \\
\text { paciente }\end{array}$ & 3,87 & $\begin{array}{l}\text { Necesidades del } \\
\text { paciente }\end{array}$ & $\begin{array}{l}\text { No Necesidades del } \\
\text { paciente }\end{array}$ & 3,87 \\
\hline $\begin{array}{l}\text { Conoce el sistema de } \\
\text { gestión de calidad }\end{array}$ & $\begin{array}{l}\text { no_Conoce el sistema } \\
\text { de gestión de calidad }\end{array}$ & 2,84 & $\begin{array}{l}\text { Recuerda el decálogo } \\
\text { de humanización }\end{array}$ & Ns/Nr_decálogo & 2,37 \\
\hline
\end{tabular}

Fuente: elaboración propia. 
En el clúster número uno se encuentran agrupados los servidores que conceden importancia a las necesidades del paciente en la Humanización, no consideran importantes las categorías de buen trato y valores y no conocen el sistema de gestión de calidad.

En contraposición tenemos el clúster número dos que agrupa los servidores que manifiestan la importancia de las características del buen trato, los valores y cualidades necesarias, sin embargo no ven la importancia de las necesidades del paciente y no conocen el decálogo de Humanización.

\section{Usuarios}

$\mathrm{Al}$ análisis de la información de usuarios también fue aplicado el Análisis de Correspondencias Múltiples (ACM), la cual deja ver cuáles son los grupos que existen según las características de las categorías, y da un gráfico de dichos grupos; en la Tabla 2 se resumen las características particulares de cada clúster para los usuarios.

Tabla 2. Caracterización por categorías de grupo para usuarios (Clúster)

\begin{tabular}{|c|c|c|c|c|c|}
\hline \multicolumn{6}{|c|}{ Corte "a" del árbol en dos ǵrupos (CLUSTERS) } \\
\hline \multicolumn{3}{|c|}{$\begin{array}{c}\text { Grupo: CLUSTER } 1 / 2 \\
\text { (Conteo: } 85 \text { - Porcentaje: } 25.84 \text { ) }\end{array}$} & \multicolumn{3}{|c|}{$\begin{array}{c}\text { Grupo: CLUSTER 2/2। } \\
\text { (Conteo: } 244 \text { - Porcentaje: } 74.16 \text { ) }\end{array}$} \\
\hline Etiqueta de variable & $\begin{array}{l}\text { Caracte- } \\
\text { rísticas de } \\
\text { categorías }\end{array}$ & $\begin{array}{l}\text { Prueba } \\
\text { valor }\end{array}$ & Etiqueta de variable & $\begin{array}{l}\text { Caracte- } \\
\text { rísticas de } \\
\text { categorías }\end{array}$ & $\begin{array}{l}\text { Prueba } \\
\text { valor }\end{array}$ \\
\hline $\begin{array}{l}\text { En la atención recibida, con los } \\
\text { diferentes procedimientos se tiene en } \\
\text { cuenta su comodidad }\end{array}$ & Bueno & 12,00 & $\begin{array}{l}\text { En la atención recibida, con los } \\
\text { diferentes procedimientos se tiene en } \\
\text { cuenta su comodidad }\end{array}$ & Malo & 12,41 \\
\hline $\begin{array}{l}\text { La atención recibida es cordial y } \\
\text { respetuosa a usuarios y familiares }\end{array}$ & Bueno & 11,43 & $\begin{array}{l}\text { La atención recibida es cordial y } \\
\text { respetuosa a usuarios y familiares }\end{array}$ & Malo & 11,43 \\
\hline $\begin{array}{l}\text { Se da prioridad a niños, adultos } \\
\text { mayores, obstétricos y pacientes en } \\
\text { condiciones críticas }\end{array}$ & Bueno & 10,21 & $\begin{array}{l}\text { Se da prioridad a niños, adultos } \\
\text { mayores, obstétricos y pacientes en } \\
\text { condiciones críticas }\end{array}$ & Malo & 10,07 \\
\hline $\begin{array}{l}\text { Le brindan información clara y } \\
\text { oportuna, atendiendo las inquietudes } \\
\text { manifestadas por los usuarios y su } \\
\text { familia }\end{array}$ & Bueno & 9,07 & $\begin{array}{l}\text { Le brindan información clara y } \\
\text { oportuna, atendiendo las inquietudes } \\
\text { manifestadas por los usuarios y su } \\
\text { familia }\end{array}$ & Malo & 9,07 \\
\hline $\begin{array}{l}\text { Lo tratan con igualdad, sin } \\
\text { discriminación }\end{array}$ & Bueno & 7,51 & $\begin{array}{l}\text { Lo tratan con igualdad, sin } \\
\text { discriminación }\end{array}$ & Malo & 7,55 \\
\hline $\begin{array}{l}\text { Se respeta la privacidad y dignidad de } \\
\text { los usuarios y su familia }\end{array}$ & Bueno & 7,45 & $\begin{array}{l}\text { Se respeta la privacidad y dignidad de } \\
\text { los usuarios y su familia }\end{array}$ & Malo & 7,45 \\
\hline $\begin{array}{l}\text { Protegen su información, la cual no es } \\
\text { divulgada sin su consentimiento }\end{array}$ & Bueno & 5,00 & $\begin{array}{l}\text { Protegen su información, la cual no es } \\
\text { divulgada sin su consentimiento }\end{array}$ & Malo & 5,28 \\
\hline $\begin{array}{l}\text { Desde que año utiliza los servicios del } \\
\text { hospital }\end{array}$ & $\begin{array}{l}\text { Antes del } \\
2012\end{array}$ & 3,59 & $\begin{array}{l}\text { Desde que año utiliza los servicios del } \\
\text { hospital }\end{array}$ & $\begin{array}{c}\text { Entre } 2012 \\
\text { y } 2013\end{array}$ & 4,96 \\
\hline $\begin{array}{l}\text { Conoce usted los deberes y derechos } \\
\text { que tiene como usuario }\end{array}$ & $\begin{array}{l}\text { Si conoce } \\
\text { los deberes }\end{array}$ & 2,78 & $\begin{array}{l}\text { Conoce usted los deberes y derechos } \\
\text { que tiene como usuario }\end{array}$ & $\begin{array}{l}\text { No conoce } \\
\text { los deberes }\end{array}$ & 2,78 \\
\hline Sexo & Femenino & 2,38 & Sexo & Masculino & 2,38 \\
\hline
\end{tabular}

Fuente: elaboración propia. 


\section{Percepción de los usuarios}

A la información reunida de los usuarios le fue aplicado un indice general de componentes pincipales cuyos valores oscilan entre cero y cien, siendo cero el valor más bajo e indica que la percepcion del servicio es mala; los valores cercanos a cien indican que la percepción es buena. Este índice permite conocer a nivel general mediante un número cuál es la percepción del usuario según las caracteristicas evaluadas y da un peso a cada variable según su importancia en la prueba. Los resultados se presentan en las Tablas 3 y 4.

Tabla 3. Valor del Índice de Componentes Principales y Valor Ponderado de las Variables para la percepción del usuario de la humanización en el servicio de urgencias

\begin{tabular}{|l|c|}
\hline \multicolumn{1}{|c|}{ Variable } & Valor ponderado \\
\hline $\begin{array}{l}\text { La atención es cordial y respetuosa a } \\
\text { usuarios y familiares }\end{array}$ & $15,8 \%$ \\
\hline $\begin{array}{l}\text { Le brindan información clara y oportuna } \\
\text { respondiendo las inquietudes manifestadas } \\
\text { por los usuarios y su familia }\end{array}$ & $14,2 \%$ \\
\hline $\begin{array}{l}\text { Se respeta la privacidad y dignidad de los } \\
\text { usuarios y su familia. }\end{array}$ & $14,4 \%$ \\
\hline Trato con igualdad, sin discriminación & $15,0 \%$ \\
\hline $\begin{array}{l}\text { Protegen su información, la cual no es } \\
\text { divulgada sin su consentimiento }\end{array}$ & $9,4 \%$ \\
\hline $\begin{array}{l}\text { Se da prioridad a niños, adultos mayores, } \\
\text { obstétricos y pacientes en condiciones } \\
\text { citicas }\end{array}$ & $14,9 \%$ \\
\hline $\begin{array}{l}\text { En la atención recibida, con los diferentes } \\
\text { procedimientos se tiene en cuenta su } \\
\text { comodidad }\end{array}$ & $16,2 \%$ \\
\hline Índice General de componentes principales & $79,3 \%$ \\
\hline
\end{tabular}

Fuente: elaboración propia a partir de los resultados del procesamiento en el programa estadístico SPSS.
Tabla 4. Convergencias en los obstáculos identificados por servidores y usuarios del servicio de urgencias del Hospital Fontibón

\begin{tabular}{|c|}
\hline Se requiere más personal \\
\hline Más atención a pacientes \\
\hline Prontitud en los exámenes \\
\hline Uso del celular en horas laborales \\
\hline Falta de comunicación médico-paciente \\
\hline
\end{tabular}

Fuente: elaboración propia.

La falta de atención se relaciona con la falta de personal ya que la calidad y tiempo de la atención médica se ve afectada por la falta de personas, dado que el servidor debe atender a todos los pacientes disminuyendo las características de la consulta antes mencionadas.

La demora en la realización de los exámenes, o en la entrega de los resultados genera en algunos casos que el paciente deba permanecer mucho más tiempo en el servicio de urgencias, generando congestión en el servicio ya que ocupa un lugar que podría ser para otra persona que está en la sala de espera.

El uso del teléfono celular en horas laborales, fue mencionado tanto por servidores como por usuarios como un obstáculo para la humanización del servicio de urgencias, ya que esta práctica hace que el servidor se desconcentre de su trabajo, además que el tiempo dedicado al celular es tiempo que le quitan a los usuarios que están esperando a ser atendidos, y teniendo en cuenta la naturaleza del servicio de urgencias, los usuarios si bien son conscientes de la prioridad y los turnos, esperan una pronta atención.

La falta de comunicación médico-paciente entorpece los procesos de atención tanto en tiempo como en calidad generando tiempos diferentes en consulta, tensión e incomodidad entre las partes. 


\section{Matriz de correlación}

A partir del análisis documental se realizó la matriz de correlación, elaborada con un enfoque de procesos, por lo tanto se establecieron cuatro aspectos para su elaboración:

El contexto necesario; porque en él se establecen los pilares normativos y marco de acción de la institución frente a su entorno y las necesidades a cubrir en su zona de acción, plasmado en la planeación estratégica.

Las entradas; contempladas aquí como los lineamientos que se establecen y reformulan periódicamente según los periodos de gobierno y las propuestas desarrolladas con sus respectivos enfoques, es así como se incluye el eje estratégico "Una ciudad que supera la segregación y la discriminación : el ser humano en el centro de las preocupaciones humanas".
Los procesos; desde la perspectiva del modelo NTCGP 1000 se tiene como principio un enfoque en procesos, para que las organizaciones se gestionen de manera eficaz teniendo que determinar actividades que se interrelacionan. Tomando el mapa de procesos del Hospital, en este apartado se identificaron aquellos procesos del Hospital que están inmersos en la planificación o los efectos y resultados visibles de la humanización de los servicios de salud.

En relación con los productos; se contemplan las construcciones del Hospital en torno al tema de humanización y calidad, ya que con ellos materializa la adaptación de las normas aplicables en su contexto y con los cuales se busca la satisfacción de las necesidades de los usuarios, respondiendo a su vez al logro de los objetivos estratégicos.

Figura 4. Matriz de correlación

\begin{tabular}{|c|c|c|c|}
\hline \multicolumn{4}{|c|}{ MATRIZ DE CORRELACION } \\
\hline \multirow{5}{*}{$\begin{array}{c}\text { PLAN DE } \\
\text { DESARROLLO } \\
\text { 2012-2016 } \\
\text { BOGOTÁ } \\
\text { HUMANA }\end{array}$} & PLAN TERRITORIAL DE SAL & BOGOTÁ DISTRITO CAPITAL 2012-2016 & \multirow{5}{*}{$\begin{array}{c}\text { HOSPITAL } \\
\text { FONTIBON } \\
\text { II nivel } \\
\text { E.S.E. }\end{array}$} \\
\hline & CONTEXTO & ENTRADA & \\
\hline & $\begin{array}{c}\cdot \text { Misión. } \\
\cdot \text { Visión. } \\
\cdot \text { Valores. } \\
\cdot \text { Principios. } \\
\cdot \text { Objetivos Corporativos. } \\
\text { Normas y leyes que rigen el servicio } \\
\text { de salud en Colombia. }\end{array}$ & $\begin{array}{c}\text { - Eje estratégico: Una ciudad que supera la segregación } \\
\text { y la discriminación: el ser humano en el centro de las } \\
\text { preocupaciones del desarrollo. } \\
\text { - Plan operativo del hospital y Lineamientos } \\
\text { del servicio de urgencias. }\end{array}$ & \\
\hline & PROCESOS & PRODUCTO & \\
\hline & $\begin{array}{c}\text { - Atención al Usuario y Participación Social. } \\
\text { • Gestión del Talento Humano. } \\
\text { • Urgencias. } \\
\text { • Gestión de Calidad y Control - Gestión } \\
\text { Gerencial. } \\
\text { • Direccionamiento Estratégico y } \\
\text { Planeación }\end{array}$ & $\begin{array}{c}\text { • Política del Sistema de Gestión de Calidad. } \\
\text { • Política y Decálogo de Humanización. } \\
\text { • Código de ética y decálogo de la calidez } \\
\text { del servicio. } \\
\text { • Indicadores de cumplimiento: satisfacción en } \\
\text { la atención, humanización, Derechos y Deberes } \\
\text { conocimiento colaboradores, Derechos y Deberes } \\
\text { adherencia colaboradores, Derechos y Deberes } \\
\text { conocimiento usuarios, Derechos y Deberes adherencia } \\
\text { usuarios, quejas por trato deshumanizado. }\end{array}$ & \\
\hline
\end{tabular}

Fuente: elaboración propia. 
Según lo observado en el desarrollo de la investigación y los resultados obtenidos en los usuarios, se evidencia el trabajo realizado por el Hospital en la articulación de la Humanización en su plataforma estratégica, hecho que se hace visible a nivel documental y se demuestra en la sinergia de la Humanización con el Sistema de Gestión de Calidad del Hospital de Fontibón E.S.E. II nivel, reflejado en el conocimiento de los deberes y derechos que tienen los usuarios, mostrando así el trabajo constante en el desarrollo e inclusión del usuario en las actividad dispuestas, y su interés para la socialización de los temas referentes a Humanización de los servicios de salud, certeza que se evidencia en las mejoras en la prestación del servicio de urgencias que perciben los usuarios.

Como se demuestra en el índice de percepción de la Humanización el servicio de urgencias el cual se determinó con un valor de 79,30 \% y es indudablemente el resultado de la dedicación y entrega del personal de Urgencias. Sin embargo esto también muestra que hay un porcentaje de insatisfacción, dejando un marco de acción para la mejora.

Teniendo en cuenta esto y los resultados obtenidos en los servidores públicos se plantean los lineamientos institucionales como herramientas fundamentales para lograr mejoras a nivel de servicio y de satisfacción del usuario, como en las competencias, cultura y sentido de pertenencia de los servidores, ya que por las condiciones del servicio de urgencias los servidores están sometidos constantemente a situaciones donde es imperativo el sentido de pertenencia, la responsabilidad social personal y empresarial como la inteligencia emocional, herramientas que le facilitaran desenvolverse de manera asertiva ante las diferentes situaciones 0 actitudes del usuario o compañero de trabajo.
Tabla 5. Componente, dimensión y resultado esperado

\begin{tabular}{|c|c|c|}
\hline Componente & Dimensión & $\begin{array}{c}\text { Resultado } \\
\text { esperado }\end{array}$ \\
\hline $\begin{array}{c}\text { Responsabilidad ética } \\
\text { y social del profesional } \\
\text { en la empresa. }\end{array}$ & Profesional. & $\begin{array}{c}\text { Sentido de } \\
\text { pertenencia. }\end{array}$ \\
\hline $\begin{array}{c}\text { La pedagogía de la } \\
\text { humanización. }\end{array}$ & Aprendizaje. & $\begin{array}{c}\text { Aprendizaje y } \\
\text { aplicación de } \\
\text { conocimientos. }\end{array}$ \\
\hline Inteligencia emocional. & Comportamientos. & $\begin{array}{c}\text { El arte de la } \\
\text { influencia, las } \\
\text { emociones son } \\
\text { contagiosas. }\end{array}$ \\
\hline
\end{tabular}

Fuente: elaboración propia.

Estos tres componentes son herramientas valiosas en la materialización de la Humanización de los servicios de salud, ya que pueden ser aplicados tanto en usuarios como en servidores públicos mejorando significativamente el ambiente de trabajo para los servidores y la percepción del servicio para el usuario.

\section{CONCLUSIONES}

El Hospital Fontibón E.S.E. II nivel ha incorporado a su Programa de Humanización los elementos que aportan los lineamientos emitidos a nivel Distrital como son: el Plan territorial en salud, el Plan de Desarrollo y el Programa de humanización de los servicios de salud, que contienen los temas base para la materialización de la Humanización en los servicios de salud, documentos que se han articulado con el sistema de gestión de calidad, dando como resultado la adopción de políticas propias del Hospital, adaptadas a las necesidades de la población atendida en la localidad. 
Las acciones establecidas e implementadas por el Hospital Fontibón en el tema de Humanización son importantes para lograr la acreditación en salud, objetivo del Hospital para el año 2016 y tienen un efecto positivo sobre el sistema de gestión de la calidad de la entidad, ya que se corresponden con la satisfacción del usuario, y además permiten aplicar los principios de la norma NTCGP 1000:2009 sobre: enfoque al cliente, liderazgo, mejora continua y participación activa de los servidores. Sin embargo, a través de la evidencia encontrada en el desarrollo de este estudio, algunos aspectos de la Humanización como el principio relacionado con la participación activa de los servidores, se debe fortalecer con el fin de mantener la cultura de mejora continua y procurar el bienestar tanto de usuarios como de servidores del Hospital.

En el servicio de urgencias se deben consolidar los conocimientos y las actividades del Programa de Humanización, con el fin de lograr la adherencia de las personas y reforzar la cultura de mejora continua. Se evidencia la necesidad de formular indicadores de eficiencia contrastando así los costos y el cumplimiento de los objetivos de las actividades de Humanización del servicio, con herramientas de medición que se adapten a las características y necesidades del Hospital, para evaluar la asimilación de los saberes propios de la Humanización y que se vean evidenciados en la cultura organizacional y el clima laboral.

Dentro de los obstáculos relevantes manifestados por los usuarios (falta de atención) y por lo servidores (estado de contingencia, fallas logísticas), existen convergencias ya que los dos se relacionan con el tema de disponibilidad del talento humano de acuerdo con las competencias requeridas para la atención, agilidad y rapidez en el servicio de urgencias; en consecuencia es importante trabajar el tema de la espera en sala con el usuario, y mejorar la comunicación de los procedimientos que se deben seguir para solicitar el servicio.
Según el analisis realizado por medio del índice de componentes principales, se evidencia que el usuario le da más importancia a la comodidad que tiene durante los procedimientos realizados en la atención que recibe, seguido de la atención cordial y respetuosa, el trato con igualdad y finalmente que se de prioridad a la atención de grupos de interés ya establecidos como niños, adultos mayores, mujeres embarazadas y pacientes en condiciones criticas.

Una conclusión importante del estudio es la entrega a la entidad de los siguientes lineamientos, que resumen los resultados obtenidos y brindan la posibilidad de consolidar en el Hospital el Programa de Humanización:

\section{Responsabilidad ética y social del profesional}

Existen factores que ayudan a mejorar el grado de responsabilidad del profesional dentro de la organización. Esto ocurre cuando en el trabajador priman las competencias ético- profesionales sobre las técnico-científicas.

Es indudable que el profesional de la salud como cualquier otro debe estar técnica y científicamente capacitado, es decir, dominar ampliamente la práctica y disciplina. Sin embargo, independientemente de su grado de capacitación tecnológica existen otros aspectos que afectan directamente su carrera. Entre estos sobresalen dos: la madurez profesional y la conciencia de su responsabilidad ética y social.

En el desempeño de su trabajo, el profesional de la salud realiza dos actividades que se relacionan estrechamente, una de tipo individual y otra de carácter comunitario. De hecho la profesión se considera como "una ocupación profesional orientada a lo social" puesto que profesional es la persona que tiene encomendada, de manera habitual, una misión que cumplir en beneficio de los demás con la 
contrapartida a su favor del derecho a la justa compensación de su trabajo.

El profesional debe poner en práctica un conjunto global de competencias que le permitan ejercer la disciplina responsable frente a sí mismo y ante su sociedad. Las competencias éticas tienen que ver con los principios y valores que encarna el profesional tanto en su vida personal como profesional. Gracias a ellas este incorpora en sus juicios la consideración de problemas éticos y pondera en el papel de profesional en la sociedad; actúa para mantener firme el honor, la integridad y la dignidad de la profesión; construye su reputación profesional con mérito, es objetivo y veraz; muestra integridad personal en todo momento, honestidad y respeto a los demás; demuestra independencia y confianza en sí mismo, mantiene su propias normas de excelencia; antepone el bienestar de la comunidad a los intereses profesionales y particulares, manifiesta lealtad y fiabilidad profesional; mantiene excelentes relaciones humanas con colegas y clientes y asume la responsabilidad profesional por sus acciones (Hamburguer, 2008, pp. 319-322).

\section{La pedagogía de la humanización del trabajo}

El trabajo adquiere cada vez, con mayor velocidad, un tejido de nuevas competencias, responsabilidades y dinámicas de interacción social que lo convierten en una realidad compleja de gestión de conocimiento.

Es necesario comprender la realidad humanizante del trabajo y desarrollar un análisis y comprensión desde la pedagogía que permita comprender elementos de interacción laboral y social en el sentido y significado del trabajo.

Así, una pedagogía de la humanización del trabajo comprende por competencia aquel todo estructurado de componentes que permiten pensar, actuar, interactuar y convivir con los demás de manera pacífica y constructiva en una dinámica que hace crecer en actitud y aptitud, con unos valores esenciales que implican la realización personal, la gestión laboral y la interacción social significativas.

La pedagogía de la humanización defiende la tesis: si formamos y fortalecemos en la actitud, el amor hacia algo, si formamos sujetos con responsabilidad social y con sentido, significativos y pensamiento social, no necesitaremos preocuparnos más por la academia, por el rigor académico, este llegara con seducción.

El vacío que le preocupa superar a la pedagogía de la humanización, se centra en el entendimiento de que humanizar permite percibir personas conscientes de las consecuencias individuales y sociales de su actuar cotidiano.

Un profesional motivado y seducido por su trabajo no solo mejora su producción en cantidad y calidad sino que además mejora su calidad de vida y se convierte en un sujeto de tejido social, solidaridad consigo mismo, con los demás y con su entorno social. Convirtiendo a la organización en una comunidad que propone a sus miembros un mundo de sentido de identidad y una pertenencia, unos valores compartidos, una tarea común, un bien común. Fortaleciendo la cultura corporativa pasando de la sola eficiencia a la confianza, de la cantidad a la calidad, del conflicto a la cooperación, del solo negocio a la responsabilidad (Hamburguer, 2008, pp. 329-333).

\section{Inteligencia emocional: el arte de la influencia, las emociones son contagiosas}

El término "inteligencia emocional" se refiere a la capacidad de reconocer nuestros propios sentimientos y los ajenos, de motivarnos y de manejar 
bien las emociones, en nosotros mismos y en nuestras relaciones. Describe aptitudes complementarias, pero distintas, de la inteligencia académica, las habilidades puramente cognitivas medidas por el coeficiente intelectual (C.I.).

La inteligencia emocional es también definida en función de la capacidad de monitorear y regular los sentimientos propios y ajenos y de utilizar los sentimientos para guiar el pensamiento y la acción.

Sin embargo, se ha adaptado un modelo en una versión útil para entender la importancia que tienen estos talentos en la vida laboral, esta adaptación incluye cinco aptitudes básicas emocionales y sociales:

- Conocimiento de uno mismo. Saber que se siente en cada momento y utilizar esas preferencias para orientar nuestra toma de decisiones; tener una idea realista de nuestras habilidades y una bien basada confianza en uno mismo.

- Autorregulación. Manejar las emociones de modo que faciliten la tarea entre manos, en vez de estorbarla; ser escrupulosos y demorar la gratificación en pos de los objetivos; recobrarse bien de las tensiones emocionales.

- Motivación. Utilizar nuestras preferencias más profundas para orientarnos y avanzar hacia los objetivos, para tomar iniciativas y ser muy efectivos y para preservar frente a los contratiempos y las frustraciones.

- Empatía. Percibir lo que sienten los demás, ser capaces de ver las cosas desde su perspectiva y cultivar la afinidad con una amplia diversidad de personas.
- Habilidades sociales. Manejar bien las emociones en una relación e interpretar adecuadamente las situaciones y las redes sociales; interactuar sin dificultad, utilizar estas habilidades para persuadir y dirigir, negociar, y resolver disputas, para la cooperación y el trabajo en equipo.

El arte de la influencia requiere manejar con efectividad las emociones ajenas. Los trabajadores estelares son diestros en la proyección de señales emocionales, lo cual los convierte en potentes comunicadores, capaces de dominar a un público. En pocas palabras, los convierte en líderes.

Las emociones son contagiosas, todas estas habilidades aprovechan una realidad primaria: cada uno influye en el estado de ánimo de los demás. Es perfectamente natural influir en el estado emocional de otro, para bien o para mal; contagiar las emociones sucede constantemente, como una especie de virus social. Este intercambio emocional constituye una economía interpersonal invisible, parte de todas las interacciones humanas, pero habitualmente es tan sutil que no se lo percibe.

La transmisión con tanta facilidad de los estados de ánimo, se debe a que pueden ser señales vitales para la supervivencia. Nuestras emociones nos indican en qué concentrar nuestra atención, cuándo prepararnos para actuar. Son captadores de atención, que operan como advertencias, invitaciones, alarmas, etc., se trata de mensajes potentes que trasmiten información crucial sin poner necesariamente datos en palabras. Según Daniel Goleman (2007), las emociones son un método de comunicación híper-eficiente.

La economía emocional es la suma total de los intercambios de sentimientos entre nosotros. De manera sutiles (o no tanto) todos nos hacemos sentir un poquito mejor (o mucho peor) como parte de cualquier contacto que tengamos; cada encuentro se puede evaluar según una escala que va desde lo emocionalmente 
toxico a lo nutritivo. Aunque esta operación es en su mayor parte invisible, esta economía puede brindar inmensos beneficios a una empresa 0 al tono de vida dentro de la organización (Goleman, 2007)

\section{REFERENCIAS}

Fierro, J. (2010). Análisis estadístico univariado, bivariado y variables control(pp. 1-16). Recuperado de http://chitita.uta.cl/cursos/2012-1/0000104/ recursos/r-25.pdf

Giardina, B. (1967). Manual de estadística. Primera publicación México D.F.: Compañía Editorial Continental S.A.

Goleman, D. (2007). Inteligencia emocional en la empresa. Buenos Aires: Ediciones B Argentinas S.A..

Hamburguer, Á. (2008). Humanización de la empresa, Hacia una ética aplicada en las organizaciones. Primera edición, editorial Bonaventuriana.

Parsons, T. (1966). Estructura y proceso en las sociedades modernas. Sistema del funcionalismo estructural. Madrid: Instituto de estudios modernos.
Quaglino, M. y Pagura, J. (1998). Una propuesta para algunas aplicaciones de análisis de correspondencias múltiples (pp. 249-257). Rosario, Argentina: Universidad Nacional de Rosario, Terceras Jornadas Investigaciones en la Facultad de Ciencias Económicas y Estadística, Instituto de investigaciones Técnicas y Aplicadas.

Universidad Nacional Abierta y a Distancia-UNAD. (2014a). Lección 2. Análisis univariado. Recuperado de http://datateca.unad.edu.co/contenidos/401533/2014-1/modulo2014/leccin_2_anlisis_univariado.html

Universidad Nacional Abierta y a Distancia-UNAD. (2014b) Lección 3. Análisis Bivariado. Recuperado de http://datateca.unad.edu.co/contenidos/401533/2014-1/modulo2014/leccin_3_anlisis_bivariado.html

Universidad Nacional Abierta y a Distancia-UNAD. (2014c). Lección 4. Análisis multivariante. Recuperado de http://datateca.unad.edu.co/contenidos/401533/2014-1/modulo2014/leccin_4_anlisis_multivariante.html 